\title{
Suppression of visible persistence as a function of spatial separation between inducing stimuli
}

\author{
VINCENT DI LOLLO \\ University of Alberta, Edmonton, Alberta, Canada \\ and \\ JOHN H. HOGBEN \\ University of Western Australia, Nedlands, Australia
}

\begin{abstract}
In the first of two experiments, suppression of the visible persistence of a temporally leading stimulus by a trailing stimulus was studied as a joint function of stimulus onset asynchrony (SOA) and of spatial proximity between the two stimuli. In agreement with earlier work, temporal integration between the two successive stimuli was progressively impaired as SOA was increased. In addition, degree of suppression of visible persistence was found to increase markedly with proximity. This confirms the outcome of earlier research that employed trains of successive stimuli seen in apparent motion. However, the present finding suggests that neither a seriated mode of display nor the appearance of coherent motion are necessary for the suppressive effect of spatial proximity. The results favor an account based on inhibitory interactions, rather than one based on spatiotemporal summation. Further predictions from inhibition theory-bearing on the luminance of the component stimuli-were tested and confirmed in a second experiment.
\end{abstract}

It is known that a visual stimulus displayed for a brief interval (say, $10 \mathrm{msec}$ ) remains visible for a short period after its termination. The period of additional visibility, known as visible persistence, has been estimated at $100-150 \mathrm{msec}$. Initially visible persistence was likened (by, e.g., Neisser, 1967) to the contents of a sensory store that is charged rapidly by the external stimulus and begins to discharge as soon as the external stimulus is turned off. However, this analogy did not prove useful in accounting for later results, notably the twin findings that duration of visible persistence is negatively related to both the intensity and the duration of the inducing stimulus (see Coltheart, 1980, for a review).

An alternative approach, suggested by Di Lollo (1980), is to regard visible persistence as an independent neural response of finite duration. This hypothesis assumes that the onset of a novel visual stimulus produces a chain of processing events within the visual system, and that duration of visible persistence corresponds to the duration of an early phase of such processing activity. According to this hypothesis, visible persistence is time-locked to the onset-rather than to the termination-of the inducing stimulus. That is, once started by the onset of a new stimulus, visible persistence is held to endure to the end of the initial period of neural activity whether or not the

This research was supported by Grant 8515045 from the Australian Research Grants Scheme to John Hogben, and by Grant A6592 from the Natural Sciences and Engineering Research Council of Canada to Vincent Di Lollo, whose mailing address is: Department of Psychology, University of Alberta, Edmonton, Alberta T6G 2E9, Canada. We wish to thank Walter Bischof and Charles Bourassa for commenting on an earlier version of this article. inducing stimulus remains on view. Congruent with this approach, it is assumed that temporal integration between two successive displays will occur to the extent to which the early neural responses produced by the two stimuli overlap temporally.

It has been shown that the high-spatial-frequency contents of a display produce longer lasting visible persistence than do the lower spatial frequencies (Di Lollo \& Woods, 1981; Meyer \& Maguire, 1977). Thus, it appears that persistence is a more prominent attribute of processing in the high- than in the low-spatial-frequency visual channels. A possible neurophysiological correlate of persistence at an early phase of visual information processing has been suggested recently by Duysens, Orban, Cremieux, and Maes (1985). A more detailed description of the processing hypothesis has been presented elsewhere (Di Lollo, 1980; Di Lollo \& Bourassa, 1983).

The experimental evidence-reviewed by Coltheart (1980)-shows convincingly that visible persistence is a dependable and robust response. Yet it is equally evident that not all brief stimuli can produce visual sensations that last as long as $150 \mathrm{msec}$. Indeed, if this were the case, an object in motion would be seen as trailing a wake of smear whose length would correspond to the distance traveled in 100-150 msec. The fact that no such smear is observed in ordinary photopic viewing has prompted a search for the factors that underlie attenuation of visible persistence. A major factor, identified reliably in several experimental paradigms, is the spatial proximity of temporally successive stimuli (Burr, 1980; Di Lollo \& Hogben, 1985; Dixon \& Hammond, 1972; Farrell, 1984; Hogben \& Di Lollo, 1985). Persistence becomes 
progressively shorter as the spatial separation between successive elements in a stimulus train is decreased.

A limitation to the generality of this finding is imposed by the sequential mode of presentation employed in all the studies mentioned above. In every case, the display consisted of a train of stimuli (points or lines) shown briefly through a series of successive locations so as to produce the impression of motion. From these studies, it is not possible to say whether the role of spatial proximity in the attenuation of visible persistence depends critically on the perception of coherent motion, or whether the same effects can be obtained with displays that do not employ trains of sequential stimuli seen in smooth motion.

This issue has conceptual as well as empirical significance in that it qualifies the generality of the principles that may be used to account for the role of spatial proximity in the attenuation of visible persistence. Notably, it bears on the issue-raised by Burr (1980) and elaborated below- of whether suppression of visible persistence requires the prior activation of visual mechanisms that underlie perception of motion.

The present work examined attenuation of visible persistence as a function of the major factor identified as critical in previous research: spatial proximity of stimuli. However, in place of a train of stimuli in apparent motion, the display consisted of only two successive frames comprising two aggregates of points chosen randomly from a source matrix, as described below.

\section{EXPERIMENT 1}

Duration of visible persistence was estimated in the present work by a variant of the method of temporal integration of form. Frequently used for this purpose (e.g., Eriksen \& Collins, 1967), this method entails synthesis of a pattern whose parts are displayed discretely in rapid succession. The basic display consisted of a $5 \times 5$ square matrix of points flashed on the screen of an oscilloscope. One of the 25 points, chosen randomly on each trial, was not shown. The observer's task was to identify the location of the missing point. Observers find this task undemanding and engaging; under optimal viewing conditions, the incidence of errors is very low.

To study duration of visible persistence, the 24 points of the incomplete matrix were displayed in two successive frames of 12 points each, chosen differently on each trial. Viewed singly, each frame appeared as a random aggregate of 12 points; however, when shown simultaneously, the two frames appeared as a matrix of points with the missing element easily detectable. The two frames were separated by a variable temporal interval (interstimulus interval, ISI) during which the screen remained blank. As shown in earlier work with a similar paradigm (e.g., Hogben \& Di Lollo, 1974), integration of the two frames is progressively impaired as duration of ISI is increased. It is found that at very short ISIs (e.g., $10 \mathrm{msec}$ ) there is complete integration of the two sets of points, which are seen as a synchronous matrix. Under these con- ditions, it can be inferred that the visible persistence of the first stimulus is sufficiently long to bridge the temporal gap between the frames. However, at longer ISIs (e.g., over $200 \mathrm{msec}$ ), the display is seen as a sequence of two discrete sets of points, and the experimental task becomes impossible. An index of the duration of visible persistence is provided by the maximum duration of ISI at which the temporal integration task can still be performed.

\section{Method}

Observers. One of the authors (J.H.H.) and a male experimental assistant (R.D.B.) served as observers. Both had normal or corrected-to-normal vision.

Visual display. The stimuli were displayed on a Hewlett-Packard 1333A oscilloscope, equipped with P15 phosphor and run under the control of a PDP-11/34A digital computer. At the viewing distance of $57 \mathrm{~cm}$, one side of the $8 \times 8 \mathrm{~cm}$ display surface subtended a visual angle of $8^{\circ}$. Screen illumination was provided by a pair of shielded 15-cm Sylvania F4T5CW fluorescent tubes located to the front and sides of the display surface, so as to produce an average luminance of $0.3 \mathrm{~cd} / \mathrm{m}^{2}$, as measured by a Spectra Spotmeter.

The display consisted of 24 of the 25 points defining a $5 \times 5$ square matrix plotted in the center of the screen. The luminous intensity of the display was kept constant from day to day, and was defined with reference to a calibration test patch. The patch consisted of 784 points plotted in a $28 \times 28$ square that covered an area of approximately $4.2 \times 4.2 \mathrm{~mm}$ on the display surface. During calibration, the screen was in total darkness and the test patch was plotted continuously at a rate of $100 \mathrm{~Hz}$, each point being refreshed once every $10 \mathrm{msec}$. The intensity gain of the oscilloscope was adjusted so as to yield a reading of $30 \mathrm{~cd} / \mathrm{m}^{2}$ as measured through the $1^{\circ}$ luminance probe of a Spectra Spotmeter. This calibration procedure was carried out daily during the course of the experiment.

Luminous intensity of the points could be varied through a 12bit digital-to-analog converter on the $z$-axis of the oscilloscope, and/or by varying the frequency of intensification of the points. In the present work, the 12 points in each frame were intensified eight times (for a total duration of $1 \mathrm{msec}$ per frame) at a $z$ level that brought the display to approximately $2.0 \mathrm{log}$ units above threshold level, which had been measured in a separate psychophysical procedure.

Design and Procedure. The observer sat in a semi-dark room facing the display surface at a distance of $57 \mathrm{~cm}$ set by a headrest. Four fixation dots defined a square area, of approximately $3^{\circ}$ on a side, in the center of the display surface. All displays were plotted in the center of the square area. Observers viewed the display binocularly with natural pupils, and initiated a trial by pressing a hand-held pushbutton. Upon a buttonpress, 24 of the 25 matrix points were displayed in two successive frames of 12 points each, separated by a temporal interval (ISI) during which the screen was blank. Taken together, the points in the two frames formed a $5 \times 5$ square matrix with one element missing. The exposure duration of each frame was $1 \mathrm{msec}$. The observer's task was to identify the location of the missing element by naming its row and column coordinates within the matrix. A headphone-microphone set allowed communication between the observer and the experimenter who entered the response on the computer terminal. The 12 points in each frame were chosen randomly on every trial from the 25 -point pool. Therefore, the location of the missing element varied randomly from trial to trial.

Two factors were varied systematically in this experiment: interpoint separation and SOA (stimulus onset asynchrony: the time elapsing between the onsets of the two frames). Each SOA was composed of the duration of the first frame $(1 \mathrm{msec})$ plus the ISI. There 
were five durations of SOA: $20,27,34,41$, and $48 \mathrm{msec}$. The five SOAs were combined with four interpoint separations to yield a total of 20 experimental conditions. The levels of interpoint separation were established separately for the two observers as follows: preliminary trials were run at a fixed SOA of $20 \mathrm{msec}$ to find the minimum interpoint separation at which performance was still virtually errorless; this was found to be $6 \mathrm{~min}$ for Observer R.D.B., and $9 \mathrm{~min}$ for J.H.H. The remaining three levels were set by cumulative increments of $3 \mathrm{~min}$. Thus, the interpoint separations were $6,9,12$, and $15 \mathrm{~min}$ for R.D.B., and $9,12,15$, and $18 \mathrm{~min}$ for J.H.H. These were center-to-center angular separations between any two adjacent points within the 25 -point matrix. The diameter of one point was approximately $1.5 \mathrm{~min}$. The corresponding visual angles subtended by the total matrix were $24,36,48$, and $60 \mathrm{~min}$ for R.D.B., and 36, 48, 60, and $72 \mathrm{~min}$ for J.H.H.

An experimental session consisted of 100 randomly ordered trials comprising 20 presentations of the dot matrix at each of the five SOAs. Interpoint separation remained fixed within a given session. The 100 trials occurred in a different random order for each session and were completed within $10 \mathrm{~min}$. Interpoint separation varied haphazardly between sessions, with the limitation that there be five sessions at each of the four levels of interpoint separation. Thus, each observer was tested for a total of 100 trials in each of the 20 combinations of SOA and interpoint separation.

\section{Results}

Figure 1 shows the percentage of errors made by the two observers at each combination of SOA and interpoint separation. It is immediately apparent that both variables had a powerful effect on performance. Percentage of errors increased both as SOA was lengthened and as interpoint separation was diminished.

It must be noted that the interpoint separations in Figure 1 specify the minimum separations between adjacent points in the complete 25-point matrix. Clearly, this does not imply that every point in the leading frame was followed by a point in the second frame at the specified separation: as stated above, the configuration of the 12 points in each frame was changed randomly on every trial, so it was possible for clusters of two or more adjacent points to appear within one frame. What is ensured in this procedure is, first, that no temporally leading point is followed by a trailing point at a spatial range closer than specified and, second, that the average spatial separation between temporally successive points increases as interpoint separation is increased.

Phenomenologically, the appearance of the displays changed considerably with variations in both independent variables. At the briefest SOA ( $20 \mathrm{msec})$, all matrix points were seen simultaneously with no evidence of a temporal gap. At this SOA, performance was virtually errorless even at the smallest interpoint separation, suggesting that acuity was not a consideration. As SOA was increased, the matrix appeared to contain not one, but several missing elements from which the observer had to choose his response. The number of apparently missing elements was greatest when the SOA was long and the interpoint separation small. The display was never seen as two discrete events separated by a temporal gap; rather, at the combinations of the independent variables at which most errors were made, the stimulus was seen as a matrix of points, some of which appeared to melt away before the display ended. Notably, coherent directional motion was never observed: the predominant impression was not one of motion, but one of selective disappearance of individual matrix elements.

A related question can now be asked: Did the points that were seen to vanish prematurely belong preponderantly to the leading or to the trailing frames of each display? In the present paradigm, an error occurred when a matrix point that had actually been plotted was incorrectly named as missing. The scoring procedure checked whether that point had been displayed in the first or in

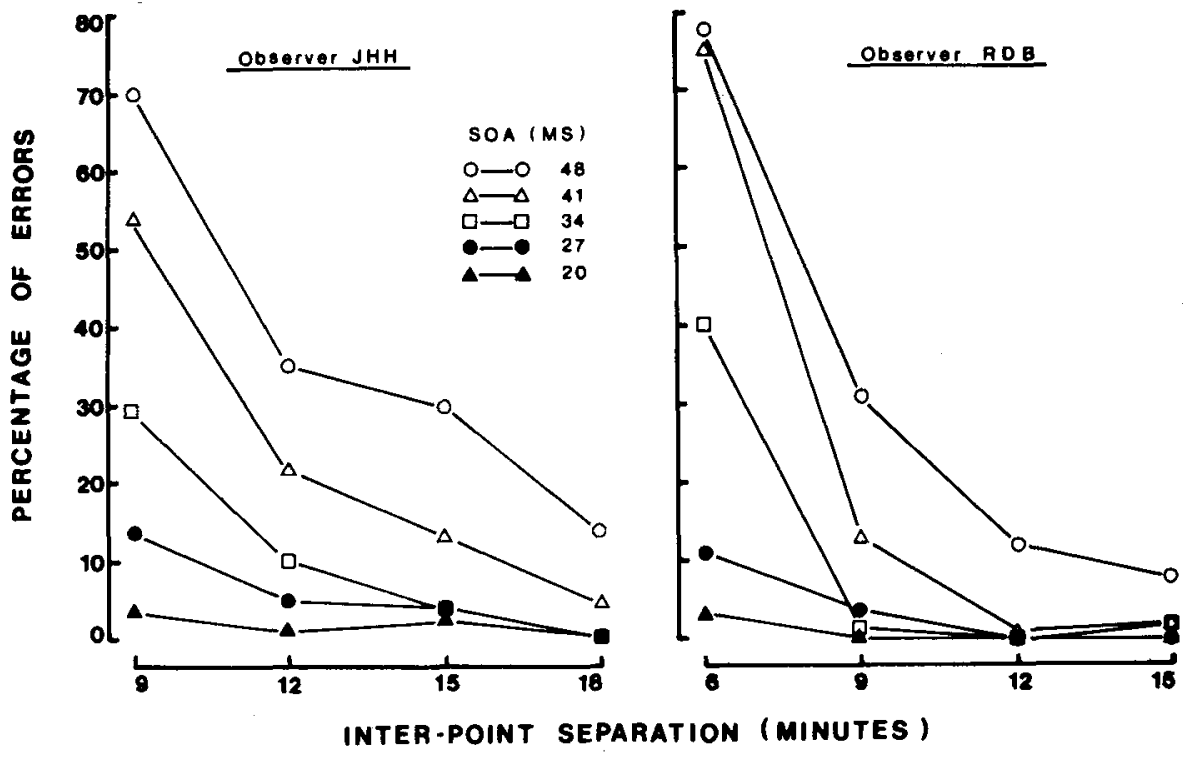

Figure 1. Percentages of errors made in Experiment 1, in relation to spatial proximity between adjacent matrix points, and SOA between the two successive frames. 
the second frame, and kept separate records of the two classes of errors for each of the 20 experimental conditions. The percentage of errors made with respect to points shown in the first frame was $\mathbf{9 2 . 1}$ for J.H.H. and $\mathbf{9 2 . 0}$ for R.D.B. These percentages remained reasonably stable and showed no systematic variation across levels of SOA or of interpoint separation. From these results, it is fair to assume that the points that were seen to vanish before the end of the display belonged preponderantly to the temporally leading frame. A compelling inference from this finding is that the visible persistence of the points in the leading set did not decay unimpeded; rather, the occurrence of the second set suppressed-or in some way interfered with-the persistence of the first. Broader implications of this finding are examined below.

\section{Discussion}

As shown in Figure 1, performance became progressively impaired as SOA was increased. However, at all except the briefest SOA, the degree of impairment depended markedly on interpoint separation, being most severe at the closest range. These results are entirely in agreement with those of earlier studies that found increasing attenuation of visible persistence (in the form of reduced motion smear) with increasing spatial proximity between successive elements of a stimulus train (e.g., Burr, 1980; Di Lollo \& Hogben, 1985; Farrell, 1984). It must be noted, however, that in the present work the attenuating effect was obtained with stimuli comprising only two successive displays that did not produce the impression of coherent motion.

There are two potential accounts of the role of stimulus proximity in the attenuation of visible persistence. One is based on spatial summation (Burr, 1980, 1984; Burr, Ross, \& Morrone, 1986), the other on inhibitory interactions (Breitmeyer, 1984; Breitmeyer \& Ganz, 1976).

According to Burr, inhibition plays no direct part in the attenuation of visible persistence (or, equivalently, in the attenuation of motion smear). Rather, two sets of visual mechanisms are postulated: one (which, for convenience, we shall label " $T$ "') is attuned to low spatial and high temporal frequencies, and is free from visible persistence; the other (which we shall label " $S$ ") is attuned to high spatial and low temporal frequencies, and is characterized by the presence of visible persistence. The mechanism by which persistence is avoided in the T system is spatial summation, whereby energy from temporally leading stimuli accrues with that of temporally trailing stimuli. At the initiation of a motion sequence (whether real or stroboscopic), a stimulus activates only the $\mathbf{S}$ mechanisms thus producing visible persistence. However, once smooth motion is established, the smearfree $T$ mechanisms are activated, and visible persistence is no longer seen. Thus, visible persistence results from the inappropriate activation of the $\mathrm{S}$ mechanisms by a moving stimulus.

This hypothesis can account reasonably well for the reduced visible persistence of a train of stimuli seen in coherent motion (e.g., a dot displayed briefly through a series of successive locations), and has been employed to account for metacontrast suppression in a two-stimulus sequence (Burr, 1984). However, the hypothesis cannot account completely for the present results. As currently stated, the summation hypothesis holds that visible persistence is obviated only for stimuli in smooth coherent motion. Indeed, activation of the $T$ mechanisms requires registration of a comprehensive motion sequence, not just a brief glimpse of it: As stated by Burr (1980, p. 165), "Brief glimpses of a motion sequence fail to activate [the T] mechanisms, activating instead [the S] mechanisms which, like a camera of long exposure, signal motion smear." Coherent motion was never seen-nor was it possible-within the displays employed in the present work, yet considerable suppression of visible persistence was obtained as a function of spatial proximity. In this respect, the present results agree entirely with the finding (e.g., Stoper \& Banffy, 1977) that marked metacontrast suppression can take place without perception of motion. It may be noted that, on occasion, there was some evidence of short-range incoherent (random) motion in the display (cf. Braddick, 1974). But this was not directional, coherent motion, and may be accountable in terms of the inhibition hypothesis outlined below.

Aside from the issue of coherence of motion, the summation hypothesis encounters conceptual problems in accounting for attenuation of visible persistence in any twostimulus sequence, whether it be in a metacontrast paradigm (e.g., Burr, 1984) or in the present paradigm. This is so because activation of the $T$ system requires a sequence of at least two stimuli, whereas only one stimulus (the first) is sufficient to activate the $S$ system, and thus produce visible persistence. Arrival of the second stimulus may (or may not) fire the T system but, according to theory, it has no effect on the ongoing activity of the S system. To explain attenuation of persistence, some form of hierarchical interaction between $S$ and $T$ systems needs to be postulated. For example, it could be assumed that the $\mathrm{T}$ mechanisms may have a shorter latency (a wellsupported assumption; see Breitmeyer, 1984) and that it takes precedence in determining what is seen. Alternatively, it may be assumed that activity in the $T$ system suppresses ongoing activity (i.e., visible persistence) in the $S$ system. Whatever the option, the summation hypothesis is clearly in need of elaboration if it is to account for suppression of visible persistence in two-part display sequences.

Inhibition has been employed as an explanatory basis in several models of suppression of visible persistence in metacontrast and in apparent motion (e.g., Breitmeyer \& Ganz, 1976; Matin, 1975; Weisstein, Ozog, \& Szoc, 1975). Among these, the model proposed by Breitmeyer and Ganz (1976) has shown broad heuristic value (cf. Breitmeyer, 1984) and provides a conceptual framework for an inhibition-based account of the present results.

Breitmeyer and Ganz's inhibition theory postulates essentially the same two sets of visual mechanisms as were 
proposed by Burr (1980), but emphasizes different properties. It assumes that stimulation activates two visual pathways or channels: the transient channel, which has short latency and responds optimally to low spatial and high temporal frequencies, and the sustained channel, which has longer latency and responds to high spatial and low temporal frequencies. To account for suppression of visible persistence in two-part display sequences, inhibition theory makes the specific assumption that activation of the transient channels produces a burst of activity that is briefer and has shorter latency than the corresponding activity in the sustained channels; it also assumes that a transient burst inhibits ongoing sustained activity in adjacent areas. A comprehensive account of the psychophysical and neurophysiological evidence in support of these assumptions has been provided by Breitmeyer and Ganz (1976). In brief, if two adjacent stimuli are displayed successively at an appropriate temporal interval, the fast transient activity produced by the second stimulus will inhibit the more sluggish sustained activity (i.e., the visible persistence) produced by the first.

Two salient aspects of the present results are in need of explanation. As shown in Figure 1, performance becomes progressively impaired (and, by inference, duration of visible persistence is progressively reduced) both as interpoint separation is decreased and as SOA is increased. In broad outline, inhibition theory can account for these effects on the twin grounds that strength of inhibition diminishes with distance between stimulated points, and that a minimum temporal interval-based on the difference in latency between transient and sustained pathways-must elapse before inhibitory effects can develop. The two accounts are examined below, in turn.

Breitmeyer (1984, pp. 218-219) notes that the distance at which inhibitory interactions can occur is set by the spatial extent of neuroanatomical structures such as receptive fields, both retinally and cortically. In particular, at cortical levels, transient-on-sustained inhibition should be greatest within a columnar structure and should diminish with distance between columns. Although not identifying the locus of the effects, the curves in Figure 1 may be regarded as estimates of the spatial extent of inhibitory interaction under the present viewing conditions. At a first approximation, the data suggest a spatial extent of less than $20 \mathrm{~min}$. This agrees well with similar estimates obtained in psychophysical studies of line-spread functions (e.g., Wilson, Phillips, Rentschler, \& Hilz, 1979) and of inhibitory interactions between two sequential points of light (Vrolijk \& Van der Wildt, 1985).

Before turning to an inhibition-based account of the effects of SOA, an alternative hypothesis must be considered and discounted. It may be suggested that performance deteriorated at longer SOAs not because of any suppressive effects, but because visible persistence might have decayed spontaneously as a function of time elapsed since the onset of stimulation. This hypothesis is contradicted by the data (Figure 1), which show that, at all but the briefest SOA, the number of errors ranged from relatively high to quite low, depending on interpoint sepa- ration. This dependence on a variable other than SOA is inconsistent with the option that the impairment in performance reflects merely the spontaneous decay of visible persistence; instead, it suggests an active process of suppression.

An inhibitory account of the effect of SOA can best be illustrated by direct reference to the data. For Observer J.H.H., consider the levels of performance at each of the five SOAs, given an interpoint separation of $9 \mathrm{~min}$ (Figure 1). Little or no suppression is evident at an SOA of $20 \mathrm{msec}$ because the transient burst produced by the second frame has subsided before the onset of the sustained activity produced by the first frame. Under these conditions, the sustained activity produced by the first frame remains unsuppressed and goes on to overlap temporally with that produced by the second frame, thus permitting perceptual integration of the matrix form. This sequence of events is illustrated in panel A of Figure 2.

By contrast, at an SOA of $48 \mathrm{msec}$, the sustained activity produced by the first stimulus has had time to develop before the arrival of the transient activity produced by the second frame. However, when it arrives, the transient burst inhibits the ongoing sustained activity, leaving little, if any, to be integrated with the ensuing sustained activity from the second frame. The inhibitory effect of the longer SOA is illustrated in panel B of Figure 2. At the intermediate SOAs, the levels of performance reflect varying degrees of inhibition, depending on the extent of temporal overlap between the secondframe transient burst and the subsequent first-frame sustained activity.

In a nutshell, if two successive stimuli are spatially close enough for inhibitory interactions, unimpeded temporal integration between them is restricted to SOAs that permit the transient response to the second stimulus to subside before the onset of the sustained response to the first. At longer SOAs, any ongoing first-frame sustained activity is suppressed-partially or totally-by the transient burst produced by the second frame, thus impairing the integration of the two sets of sustained activities. Invariably, it is the activity produced by the first frame that is suppressed by the onset of the second. The finding that virtually all errors in the present work arose from naming one of the points in the first frame as missing is entirely consistent with this explanation.

Even though the present account of the effect of SOA has been couched with reference to a single interpoint separation, precisely the same account applies to all interpoint separations in Figure 1. Although the absolute number of errors diminished as spatial separation was increased (reflecting the waning strength of inhibitory interactions at greater distances), the levels of performance remained ordered according to duration of SOA.

\section{EXPERIMENT 2}

According to the inhibition hypothesis, perceptual integration between two brief successive stimuli can take place to the extent to which the transient burst produced 

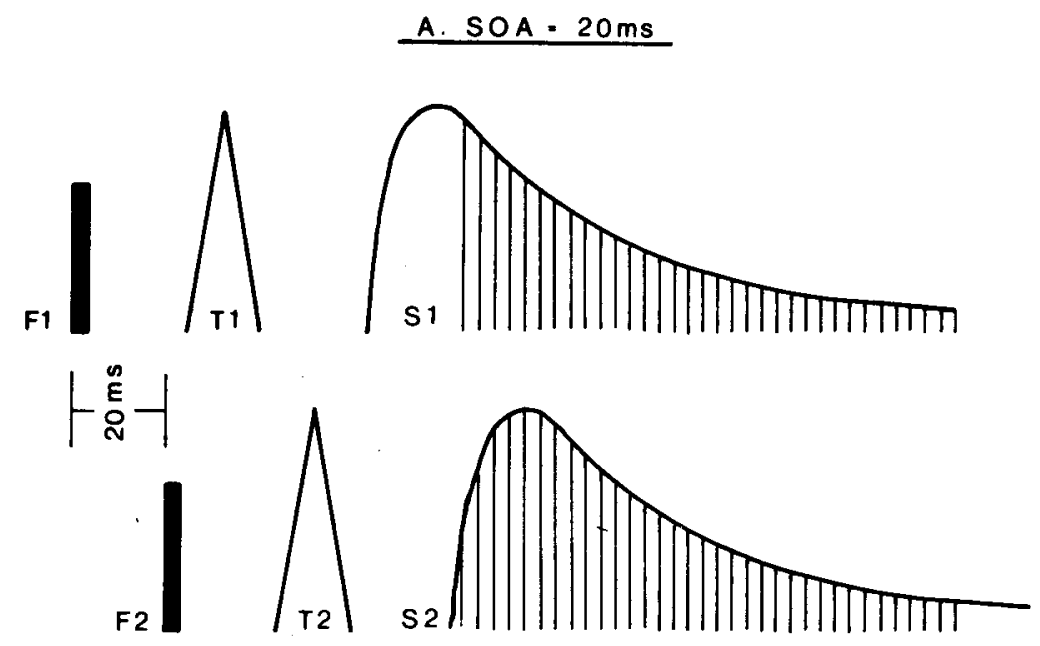

Time

B. $S O A=48 \mathrm{~ms}$
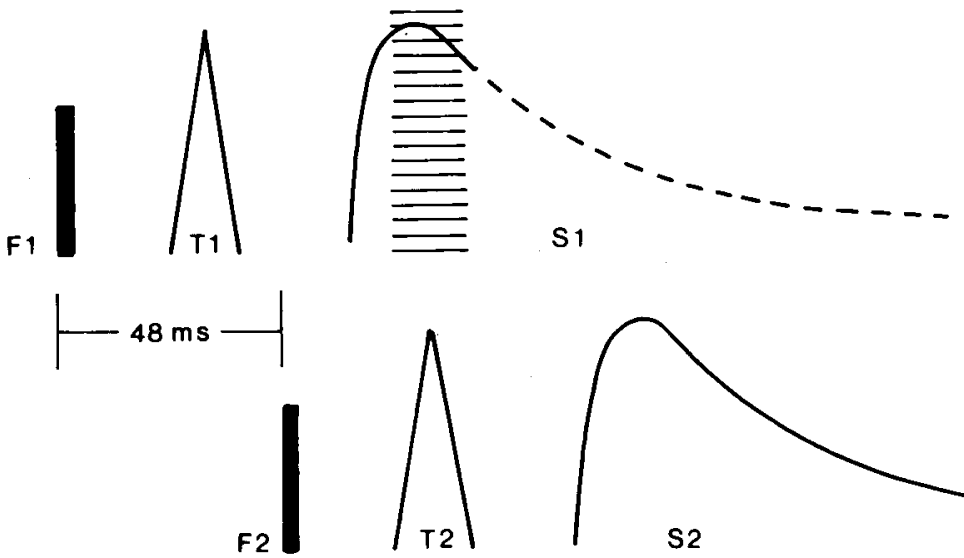

S1

Figure 2. Diagramatic representation of the inhibitory interactions between transient and sustained responses produced by a dot-matrix displayed in two successive frames at SOAs of 20 msec (panel A) and 48 msec (panel B). F1 = temporally leading frame; F2 = temporally trailing frame; T1, S1 = transient and sustained responses, respectively, produced by F1; T2, S2 = transient and sustained responses, respectively, produced by F2. Vertical hatch marks in panel $A$ indicate temporal integration between $S 1$ and S2. Horizontal hatch marks in panel B indicate inhibition by $\mathrm{T} 2$ on $\mathrm{S1}$. The segmented line in panel B indicates the temporal course of $\mathrm{S1}$, had there been no suppression by T2. (After Breitmeyer \& Ganz, 1976.)

by the second stimulus does not overlap with the ensuing sustained activity produced by the first. One way of increasing the period during which integration can occur is to increase the period that elapses between secondstimulus transient and first-stimulus sustained responses, as explained below. This was achieved in Experiment 2 by exploiting the relationship between stimulus intensity and response latency: given constant background lu- minance, a decrement in the intensity of a stimulus (i.e., a reduction in contrast) is known to produce a corresponding increment in the latencies of both transient and sustained responses (Ikeda \& Wright, 1972; Shapley \& Victor, 1978).

In terms of the inhibition hypothesis, the longer response latencies produced by the dimmer stimuli should increase the period during which temporal integration can 
occur. In practice, performance at a given SOA should yield fewer errors if the stimuli are dim than if they are bright. Specific predictions are illustrated in the two panels of Figure 3: Panel A illustrates the case of bright stimuli (short latencies), and panel B illustrates the case of dim stimuli (longer latencies).

The upper portion of Figure 3 illustrates the hypothetical sequence of events for a matrix displayed in two successive frames (F1 and F2) containing 12 bright points each. Given that the intensity is high, the latencies of both the transient (T1 and T2) and the sustained ( 11 and $\mathrm{S} 2$ ) responses are relatively short. The SOA between F1 and F2 has been set so that T2 occurs just after the onset of $\mathrm{S} 1$. Under these conditions, T2 will inhibit S1, thus preventing temporal integration between the two sustained responses.

The lower portion of Figure 3 illustrates a similar sequence of events for a matrix of dim points. The lower intensity of stimulation produces correspondingly longer latencies for both transient and sustained responses. Under these conditions (notably, at the same SOA as was used with the brighter stimuli), the inhibitory effect of
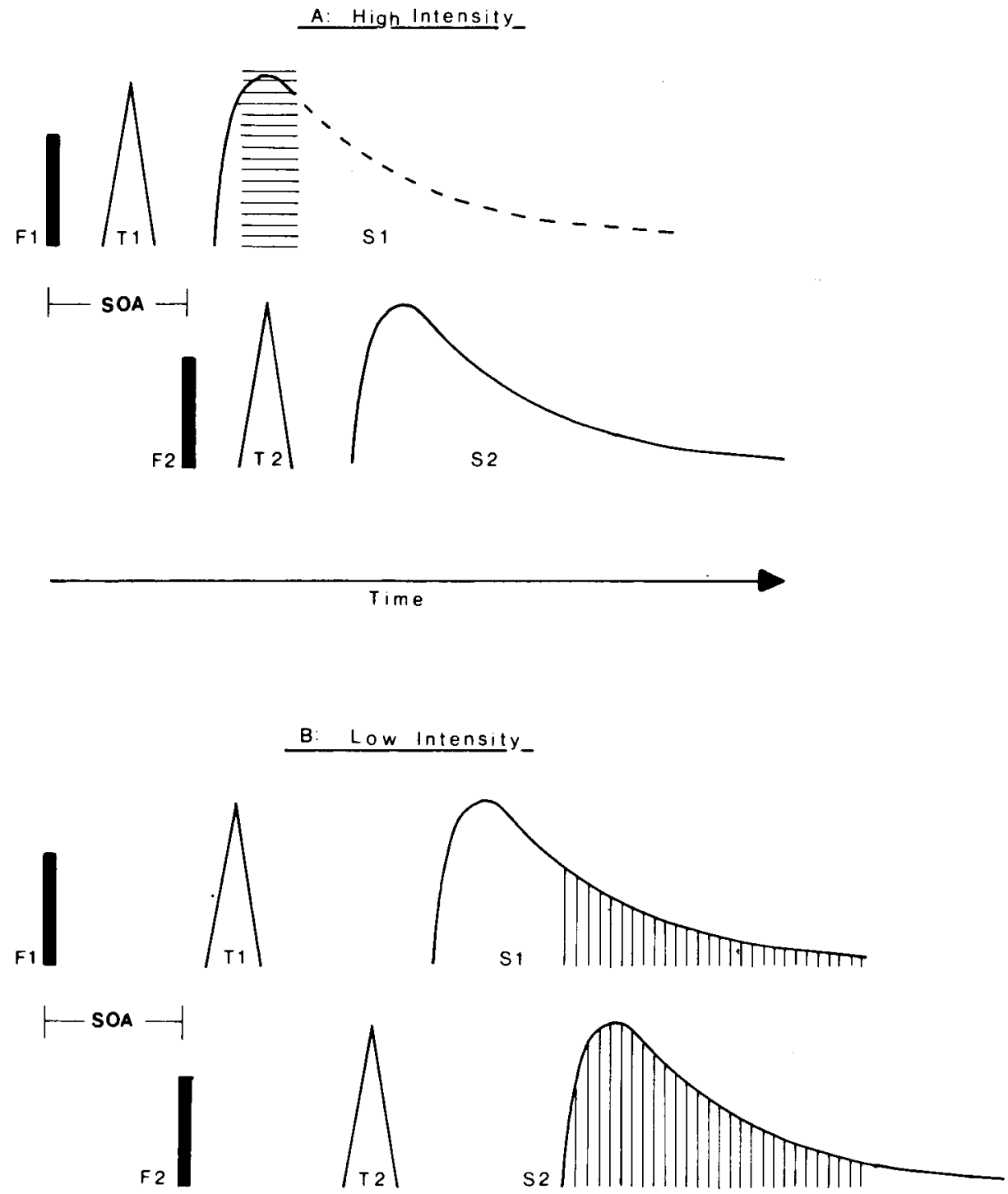

Figure 3. Diagramatic representation of the inhibitory interactions between transient and sustained responses produced by a dot-matrix displayed in two successive frames at a fixed SOA. The luminous intensity of the dots is either high (panel A) or low (panel B). F1 = temporally leading frame; F2 = temporally trailing frame; T1, S1 = transient and sustained responses, respectively, produced by F1; T2, S2 = transient and sustained responses, respectively, produced by $\mathbf{F 2}$. Vertical hatch marks in panel B indicate temporal integration between S1 and S2. Horizontal hatch marks in panel $A$ indicate inhibition by $T 2$ on S1. The segmented line in panel $A$ indicates the temporal course of S1, had there been no suppression by $\mathrm{T} 2$. The SOAs in the two panels have the same duration. The magnitudes of the inducing stimuli and of the response functions in the two panels have been normalized so as to be of the same size, despite the energy differences between high- and low-intensity stimuli. 
$\mathrm{T} 2$ subsides before the onset of $\mathrm{S} 1$, thus permitting unimpeded temporal integration between the two sustained responses.

Although transient-on-sustained inhibition is well documented (see Breitmeyer, 1984, for a review), the precise temporal and intensive relationships governing the inhibi- tory effects are not known. In the examples illustrated in Figure 3, the assumption is made that a transient burst of sufficient magnitude will inhibit completely an ensuing sustained response if the onset of the transient burst coincides with or follows the onset of the sustained activity. It is possible, of course, that the inhibitory effect

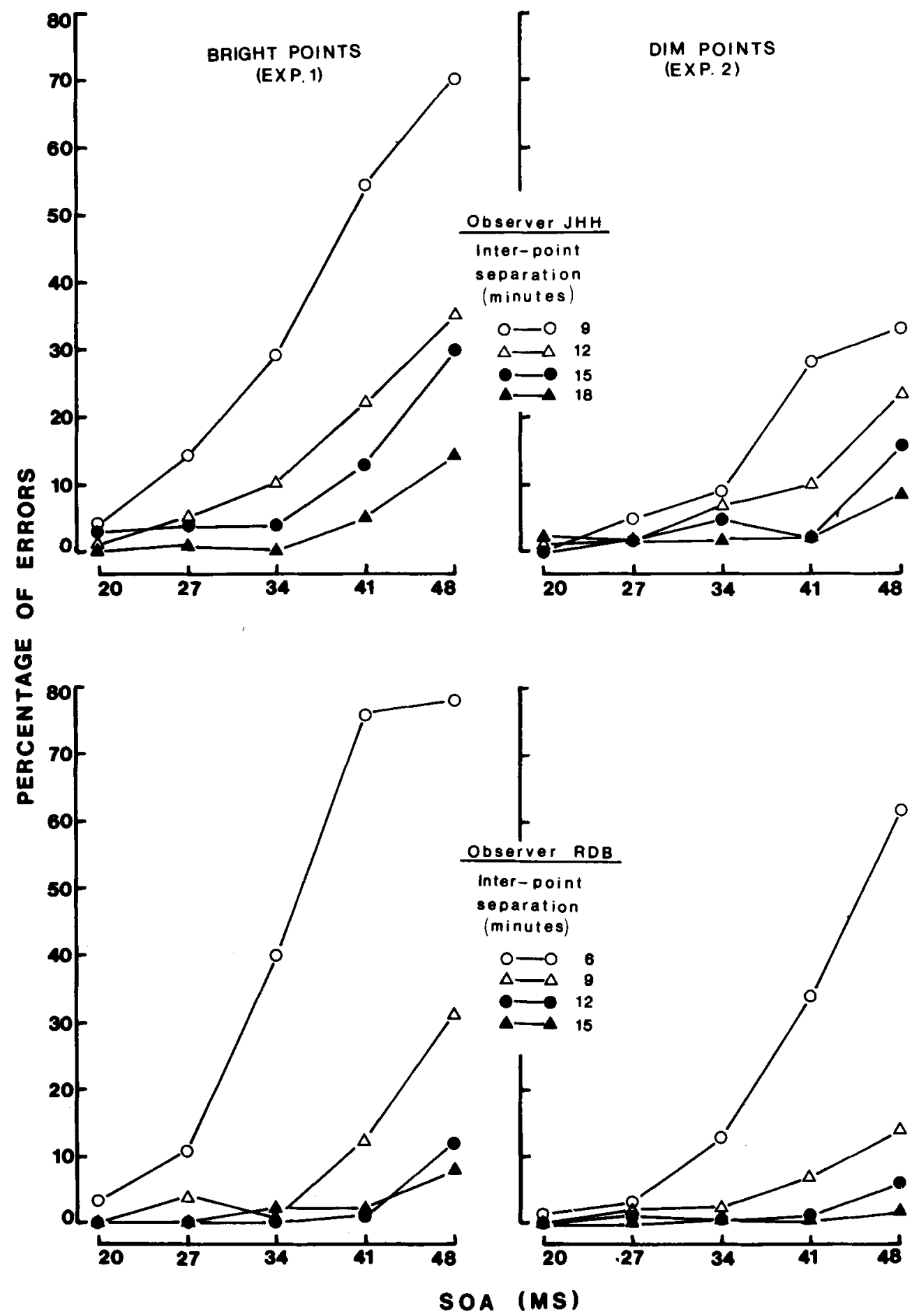

Figure 4. The percentages of errors made by the two observers in Experiment 2 (dim stimuli) are plotted in the right-side panels. The results of Experiment 1 (bright stimuli) have been replotted in the left-side panels to facilitate comparison between the outcomes of the two experiments. 
could be graded if the transient burst were weaker (as might be produced by a weaker second stimulus), or if the onset of the transient response were to precede the onset of the sustained response so as to produce only partial overlap of the two response functions. In the absence of firm parametric information, the above assumption is both plausible and minimal with respect to the predictions illustrated in Figure 3 and tested in Experiment 2.

\section{Method}

Observers, design, and procedures were the same as in Experiment 1 , with one exception: the intensity of the matrix points was $1.0 \mathrm{log}$ unit above threshold, instead of $2.0 \mathrm{log}$ units as in Experiment 1 .

\section{Results and Discussion}

The results of Experiment 2 are illustrated in Figure 4; the data from Figure 1 (Experiment 1) have been replotted in the left half of Figure 4 to facilitate comparison between the outcomes of the two experiments. It is immediately obvious that, in every instance, the two observers made substantially fewer errors when the stimuli were dim than when they were bright. In this respect, the results are consistent with the inhibitory hypothesis illustrated in Figure 3. An additional aspect of the resultsnot obvious in Figure 4 but clearly discernible when the data are plotted in the manner of Figure 1-must be noted: the spatial extent of inhibitory interactions was distinctly less among the dim stimuli of Experiment 2 than among the brighter stimuli of Experiment 1.

At the most general level, the outcome of Experiment 2 may be regarded as yet another instance of the well-known inverse intensity effect, whereby dim stimuli exhibit longer visible persistence than do brighter stimuli (cf. Coltheart, 1980). More significantly, however, the present work enables the inverse intensity effect to be encompassed within a broader conceptual framework capable of handling not only temporal but also spatial determinants of visible persistence, as well as related phenomena such as metacontrast masking (Breitmeyer, 1984).

Every curve in Figure 4 indicates that the effect of SOA is graded rather than discrete. That is, there is no evidence for a threshold value of SOA below which errors are at a minimum and above which errors suddenly reach a maximum. Instead, the incidence of errors rises steadily as SOA is increased. This pattern of results strongly suggests that the inhibitory interactions illustrated in Figures 2 and 3 are graded rather than discrete; that is, as the SOA between a temporally leading transient response and a trailing sustained response is reduced, the progressively greater overlap between the two response functions appears to produce a corresponding increment in the degree of inhibition of the transient activity. A related point must be noted: while the present results are explained readily within the conceptual framework of inhibition theory, they do not constitute a test of the specific tenets of the theory. The specific hypothesis of transienton-sustained inhibition, as well as the hypothesis of graded (as distinct from discrete) inhibitory interactions could be tested more directly by varying the magnitude of the transient response either through variations in the intensity of the second frame or through temporal ramping of its onset (see, e.g., Anstis, 1967; Krauskopf, 1980).

In contrast to the coherent account proffered by the inhibition hypothesis, the summation hypothesis appears to have no specific mechanism capable of explaining the improved performance in Experiment 2. In its present form, the hypothesis must resort to the general-and ad hocstatement that the critical period of temporal summation is longer if the stimuli are dim.

\section{CONCLUDING COMMENTS}

When permitted to run its natural course, visible persistence is known to endure at least $100-150$ msec, a relatively long duration that may produce unwanted effects such as motion smear. In ordinary viewing, persistence must be foreshortened if we are to perceive sharply defined objects. The present studies, in conjunction with earlier work, have identified spatial proximity as an important factor: duration of visible persistence is reduced as successive stimuli are brought into progressively closer spatial proximity. Notably, the suppressive effect of spatial proximity is not confined to trains of stimuli seen as in apparent motion, but occurs conspicuously with stimuli consisting of only two successive parts that produce no obvious sensation of coherent motion.

This pattern of results fits naturally within a conceptual framework of inhibitory interactions based on the distinction between transient and sustained neural networks (Breitmeyer, 1984; Breitmeyer \& Ganz, 1976). By contrast, the summation hypothesis (Burr, 1984; Burr et al., 1986) provides a less satisfactory account. In its present form, the summation hypothesis does not identify specific mechanisms capable of accounting either for suppressive effects in two-part displays, or for the effect of stimulus intensity on the critical period of temporal integration.

This is not to say that summation must be discounted as a useful explanatory basis for spatiotemporal events in vision. Indeed, considerable evidence has been adduced favoring summation as an explanatory basis for a range of perceptual phenomena such as the maintenance of figural integrity of objects in motion (Burr, 1981; Burr et al., 1986).

Rather, the present need is for identifying and describing the classes of events that are best encompassed within one or the other hypothesis. It is possible that suppressive events-such as attenuation of visible persistence or metacontrast masking - are best explained on the basis of inhibitory interactions, whereas enhancing effects-such as may be involved in maintaining the identity of moving images-are best explained in terms of summation. The option must also be considered that the two theories may be complementary rather than antagonistic. That is, both summation and inhibition may act concurrently in ordinary perceptual events: summation would act to enhance 
perception of objects in motion, while inhibition would remove unwanted residuals of stimulation-such as motion smear-that might otherwise interfere with effective functioning in a continuously changing visual environment.

\section{REFERENCES}

ANSTIS, S. M. (1967). Visual adaptation to gradual changes of intensity. Science, 155, 710-712.

Braddick, O. J. (1974). A short-range process in apparent motion. Vision Research, 13, 519-526.

BREITMEYER, B. G. (1984). Visual masking: An integrative approach. Oxford University Press: New York.

BREITMEYER, B. G., \& GANZ, L. (1976). Implications of sustained and transient channels for theories of visual pattern masking, saccadic suppression, and information processing. Psychological Review, 83, 1-36.

BURR, D. C. (1980). Motion smear. Nature, 284, 164-165.

BURR, D. C. (1981). Temporal summation of moving images by the human visual system. Proceedings of the Royal Society of London, Series B, Biological Sciences, 211, 321-339.

BURR, D. C. (1984). Summation of target and mask metacontrast stimuli. Perception, 13, 183-192.

Burr, D. C., Ross, J., \& Morrone, M. C. (1986). Seeing objects in motion. Proceedings of the Royal Society of London, Series B, Biological Sciences, 227, 249-265.

Coltheart, M. (1980). Iconic memory and visible persistence. Perception \& Psychophysics, 27, 183-228.

Di LoLLo, V. (1980). Temporal integration in visual memory. Journal of Experimental Psychology: General, 109, 75-97.

Di Lollo, V., \& Bourassa, C. M. (1983). Temporal integration following intensification of long-lasting visual displays. Vision Research, 23, 677-687.

Di Lollo, V., \& HogBen, J. H. (1985). Suppression of visible persistence. Joumal of Experimental Psychology: Human Perception \& Performance, 11, 304-316.

Di Lollo, V., \& Woods, E. (1981). Duration of visible persistence in relation to range of spatial frequencies. Journal of Experimental Psychology: Human Perception \& Performance, 7, 754-769.

Dixon, N. F., \& Hammond, E. J. (1972). The attenuation of visual persistence. British Journal of Psychology, 63, 243-254.
Duysens, J., Orban, G. A., Cremieux, J., \& Maes, H. (1985). Visual cortical correlates of visible persistence. Vision Research, 25, 171-178.

Eriksen, C. W., \& Collins, J. F. (1967). Some temporal characteristics of visual pattern perception. Journal of Experimental Psychology, 74, 476-484.

FARRELL, J. M. (1984). Visible persistence of moving objects. Journal of Experimental Psychology: Human Perception \& Performance, 10, 502-511.

HoGBEN, J. H., \& Di LoLLo, V. (1974). Perceptual integration and perceptual segregation of brief visual stimuli, Vision Research, 14, 1059-1069.

Hogben, J. H., \& Di Lollo, V. (1985). Suppression of visible persistence in apparent motion. Perception \& Psychophysics, 38, 450-460.

IKEDA, H., \& WRIGHT, M. J. (1972). Receptive field organization of 'sustained' and 'transient' retinal ganglion cells which subserve different functional roles. Journal of Physiology, 227, 769-800.

KRAUSKOPF, J. (1980). Discrimination and detection of changes in luminance. Vision Research, 20, 671-677.

Matin, E. (1975). The two-transient (masking) paradigm. Psychological Review, 82, 451-461.

MeYeR, G. E., \& MAGUIRE, W. M. (1977). Spatial frequency and the mediation of short-term visual storage. Science, 198, 524-525.

NeIsser, U. (1967). Cognitive Psychology. New York: AppletonCentury-Crofts.

Shapley, R., \&ictor, J. D. (1978). The effect of contrast on the transfer properties of cat retinal ganglion cells. Journal of Physiology, 285, 275-298.

STOPER, A. E., \& BANFFY, S. (1977). Relation of split apparent motion to metacontrast. Journal of Experimental Psychology: Human Perception \& Performance, 3, 258-277.

VRoliJK, P. C., \& VAN DER WILdT, G. J. (1985). Foveal inhibition measured with suprathreshold stimuli. Vision Research, 25, 1413-1421.

Weisstein, N., OzoG, G., Szoc, R. (1975). A comparison and elaboration of two models of metacontrast. Psychological Review, 82, 325-343.

Wilson, H. R., Phillips, G., Rentschler, I., \& Hilz, R. (1979). Spatial probability summation and disinhibition in psychophysically measured line-spread functions. Vision Research, 19, 593-598.

(Manuscript received September 12, 1986; revision accepted for publication December $8,1986$. 